Relations industrielles

Industrial Relations

\title{
Options humanistes, Éditions Économie et Humanisme, Les Éditions Ouvrières, Paris, 1968, 214 pages.
}

\section{Gérard Dion}

Volume 24, numéro 2, 1969

URI : https://id.erudit.org/iderudit/028029ar

DOI : https://doi.org/10.7202/028029ar

Aller au sommaire du numéro

Éditeur(s)

Département des relations industrielles de l'Université Laval

ISSN

0034-379X (imprimé)

1703-8138 (numérique)

Découvrir la revue

Citer ce compte rendu

Dion, G. (1969). Compte rendu de [Options humanistes, Éditions Économie et Humanisme, Les Éditions Ouvrières, Paris, 1968, 214 pages.] Relations industrielles / Industrial Relations, 24(2), 451-452.

https://doi.org/10.7202/028029ar

Tous droits réservés @ C Département des relations industrielles de l'Université Laval, 1969
Ce document est protégé par la loi sur le droit d'auteur. L’utilisation des services d'Érudit (y compris la reproduction) est assujettie à sa politique d'utilisation que vous pouvez consulter en ligne.

https://apropos.erudit.org/fr/usagers/politique-dutilisation/ 
Dans un neuvième chapitre, John Crispo et H.W. Arthurs étudient le contrepoids du pouvoir patronal (pp. 429$477)$ et proposent un projet d'accréditation des entrepreneurs.

Le professeur Gordon W. Bertram, responsable des chapitres 10 et 11 , examine d'abord les structures de négociations collectives (pp. 477-593) dans l'industrie de la construction au Canada, pour ensuite s'attaquer à la structure et aux changements de salaires (pp. 593$669)$. Nous devons cependant noter que l'étude du professeur Bertram a été effectuée sous les auspices de l'Equipe spécialisée en relations du travail d'Ottawa et par l'Association canadienne de la construction.

Après l'analyse des conventions collectives (1956-1966) présentée par Félix Quinet, Samuel Eckler aborde le sujet de la sécurité sociale (régimes d'Etat et régimes contractuels).

En guise de conclusion, H. Carl Goldenberg et John H.G. Crispo résument la nature du système de relations industrielles dans la construction pour ensuite présenter leurs recommandations sur les changements à apporter.

Cette étude des relations du travail dans un secteur industriel aussi important que la construction a une double utilité. En plus de proposer des solutions aux problèmes propres à cette industrie, elle est une contribution valable à une meilleure connaissance du système canadien de relations industrielles.

\section{Jean SEXTON}

Options humanistes, Editions Economie et Humanisme, Les Editions Ouvrières, Paris, 1968, 214 pages.

On a réuni dans cet ouvrage une série d'articles dont les principaux ont déjà été publiés dans Economie et $\mathbf{H u}$ manisme. Ces articles, fruit d'une réflexion commune des membres de l'Equipe, visent à faire le point sur ce qui a été la préoccupation fondamentale de ce mouvement de recherche et d'action fondé par le R. P. Lebret. Du même coup, les auteurs ont estimé que c'était là le meilleur moyen de rendre hom- mage à l'esprit et à la méthode à leur fondateur.

L'ouvrage est divisé en trois parties: mutation et permanence de l'humanisme; l'élaboration d'un humanisme; des options humanistes pour aujourd'hui.

On pose en premier lieu la question fondamentale de l'humanisme lui-même. Le déclin de l'humanisme classique a entraîné avec lui l'avilissement de la notion même d'humanisme. La mentalité moderne se refuse à se référer à des valeurs qui s'imposeraient de l'extérieur comme des absolus ainsi que le proposait l'humanisme classique avec son idéal transcendant et universel. II y a pourtant possibilité d'élaborer un humanisme à partir d'une nouvelle approche qui confronte l'homme avec le monde concret dans lequel il se trouve. Des mutations sont nécessaires, mais l'humanisme conserve toujours sa valeur et son actualité. Il est caractérisé par son pouvoir de contestation, il est un appel au dialogue et il est exigence d'intervention.

La seconde partie indique le cheminement de la découverte de ce nouvel humanisme qui ne peut être déduit d'une idéologie. Le point de départ doit être la réaction vitale d'un groupe humain face aux conditions concrètes de son existence. S'il existe plusieurs voies pour parvenir à l'élaboration de l'humanisme, est retenue celle qui est pratiquée ordinairement par Economie ..et Humanisme : l'enquête-participation. Puis comme tout humanisme doit aussi s'appuyer sur les disciplines qui ont l'homme pour objet, un chapitre en montre l'importance ainsi que les limites de leur apport. Enfin, à partir d'un exemple concret sont illustrées les étapes de l'élaboration de cet humanisme adapté aux exigences de notre époque.

Dans la logique de l'ouvrage, il fallait aboutir à des options pour les hommes d'aujourd'hui. Impossible de les envisager toutes. Deux sont privilégiées : celles qui concernent l'homme face aux sociétés industrielles et au problème mondial du développement. Et l'on répond au grand problème du pluralisme dans la société moderne. Partant de l'hypothèse où il est impossible à l'humanité de retrouver une unité culturelle, deux solutions peuvent régler les affaires du monde : le recours à la force où le plus 
puissant impose son point de vue ou la confrontation avec le dialogue. C'est la dernière qui est la seule raisonnable et la plus réaliste.

Options humanistes est destiné à avoir une grande influence. Grâce à la méthode particulière à Economie et Humanisme, il est enraciné dans le réel et nous repose des élucubrations philosophiques dogmatisantes à la mode qui sous prétexte de sauver l'Homme abstrait conduisent à un souverain mépris des hommes concrets. Les options qu'il propose : maitriser la création des besoins, promouvoir les responsabilités et inciter à la solidarité aideront à l'élaboration de ce que le $P$. Lebret appelait « une civilisation de la paix par l'authenticité de l'amour».

\section{Gérard DION}

The Mediator: Background, Self-image and Attitudes, Indik and Alii., Institute of Management and Labor Relations, The State University NewBrunswick, New-Jersey, 1966, 60 pages.

La médiation est un instrument essentiel dans le système américain de négociation collective. Elle a pour but de faciliter la solution des conflits et la négociation des conventions patronalessyndicales sans en tenir compte.

Le médiateur n'a aucun pouvoir de décision; sa tâche est d'amener les parties à une entente par la persuasion. Toutefois, ce rôle exige une certaine préparation que les auteurs de cette plaquette ont voulu connaître.

Cette monographie est le résultat de deux enquêtes par questionnaires, une première en 1962 et l'autre en 1964, sur les antécédents sociaux, les conditions d'emploi, les attitudes et les opinions des médiateurs. Pour les besoins de la cause, on n'a pas établi de distinction entre la médiation et la conciliation. Dans un premier temps, les auteurs décrivent brièvement l'évolution du système aux Etats-Unis. Viennent ensuite la compilation des données et une analyse sommaire des résultats.

Pierre DIONNE
Collective Bargaining Clauses, Guideline Forms for Use in Labor Negotiations, by Robert C. Knee and Robert C. Knee Jr., The W. H. Anderson Company, Cincinnati, 1967, 224 pages.

Comme son titre nous l'indique déjà assez bien, ce livre ne constitue ni un traité de la convention collective ni un manuel de négociation. Il s'agit plutôt d'un recueil présentant des clauses-types de conventions collectives de même que des conventions-types. Ce recueil constitue un instrument de travail qui pourra être utile aussi bien aux personnes engagées dans le processus de la négociation collective qu'à celles qui s'intéressent à la recherche dans ce domaine.

Les clauses présentées couvrent une grande variété de sujets (plus d'une quarantaine) allant de la sécurité syndicale aux droits de la gérance en passant par les plans de retraite, la procédure de griefs, la semaine garantie, les mesures de sécurité et d'hygiène, et bien d'autres encore.

Chaque clause est brièvement expliquée et commentée. Dans certains cas, les auteurs indiquent les liens d'interdépendance qui peuvent exister entre diverses clauses.

De plus, lorsqu'il s'agit de clauses plus complexes, on retrouve plusieurs types de clauses sur un même sujet. C'est ainsi, par exemple, que dans le cas de l'《 ancienneté 》, les auteurs ont voulu présenter trois séries de clauses-types afin de tenir compte, dans la mesure du possible, de l'influence que peut avoir sur une telle clause le caractère plus ou moins spécialisé des occupations ou des départements. Il en va de même pour un certain nombre d'autres clauses.

Enfin, le recueil présente des contrats-types, l'un dans le secteur de l'assurance-groupe, l'autre dans le secteur du transport. Cette dernière convention étant l'une des plus importantes aux Etats-Unis puisqu'il s'agit du «National Master Freight Agreement 》 et de l'un de ses compléments le «Central States Over-the-Road Agreement 》.

Les clauses des conventions collectives étant le plus souvent le résultat d'un compromis entre les parties, il est bien peu probable que les clauses présentées dans cet ouvrage puissent être 